\title{
Stepwise Layering of Helium Films on Solid Hydrogen
}

\author{
D. Cieslikowski, P. Leiderer and A. J. Dahm* \\ Fachbereich Physik, Universität Mainz, FRG
}

The mobility of surface bound electrons is monitored as helium is adsorbed on a hydrogen substrate. Maxima in the mobility occur at the completion of each helium monolayer. Up to nine maxima are observed, indicating that stepwise layering occurs for at least nine layers. A smooth variation of the mobility with further helium adsorption indicates that contimuous wetting occurs.

\section{Introduction}

The wetting behavior of helium is interesting because quantum effects may play a role and because some mechanisms responsible for nonwetting by other adsorbates can be ruled out $/ 1 /$. A number of experiments have provided evidence that helium physisorbed on various substrates, including graphite, mylar, $\mathrm{MgO}, \mathrm{Ag}$ and $\mathrm{Au}$, exhibits incomplete wetting at low temperatures $/ 2 /$. Uniform layer deposition occurs up to a substrate dependent critical thickness of one to five layers at which bulk condensation occurs. Krim and Dash $/ 1 /$ postulated that incomplete wetting is probably associated with the solidification of layers close to the substrate and with the interfacial surface energies. Recent results on vibrating graphite and platinum wires show that superfluid helium wets these substrates, confirming results of third sound experiments on graphite fibers $/ 3 /$, whereas films of normalfluid ${ }^{4} \mathrm{He}$ and ${ }^{3} \mathrm{He}$ are limited to less than thirteen layers $/ 4 /$.

We report a study of layerwise adsorption of helium on a substrate with a weaker interaction potential than previous substrates investigated. Our results imply complete wetting of helium on hydrogen, a sytem characterized by a van der Waals constant of only $20 \mathrm{~K}$ (layer) ${ }^{3}$. A more detailed report is given elsewhere $/ 5 /$.

\section{Experimental Design and Results}

Our experimental cell contains a $1 \mathrm{~mm}$ thick hydrogen substrate, grown on a glass platelet by slowly cooling from the liquid phase, and a fixed amount of helium vapor. The helium layers are adsorbed on the hydrogen from the vapor as the temperature is lowered. Electrons, deposited on the hydrogen surface from a gaseous discharge, are probed by electrodes evaporated on the lower side of the glass platelet. A small ac voltage,

Permanent address: Physics Department, Case Western Reserve University, Cleveland $\mathrm{OH} 44106$ capacitively coupled to one of the electrodes, causes a perturbation of the electron distribution and therefore produces a pick-up signal at the second electrode. The measured amplitude and phase $\varphi$ of the signal depend on both the mobility $\mu$ and the density $n$ of the electrons. To first order $\tan \varphi$ is linear in $\mu^{-1}$. The mobility is limited by scattering of the electrons by surface defects in the hydrogen crystal and from excitations in the adsorbed helium film.

Our results for $4 \mathrm{He}$ are plotted as the phase of the signal versus temperature in Fig. 1 . The amount of helium in the cell, when totally condensed, corresponds to a film thickness of ten layers. Arrows indicate the temperatures where according to a van der Waals relation completed layers are expected. The agreement with the mobility maxima is obvious.

For less helium in the cell the number of oscillations in the amplitude and phase are restricted to the total number of deposited layers. Thicker films require a higher vapor pressure in the cell and film growth commences at a higher temperature with the result that the mobility oscillations are less pronounced due to thermal excitations. An important observation in

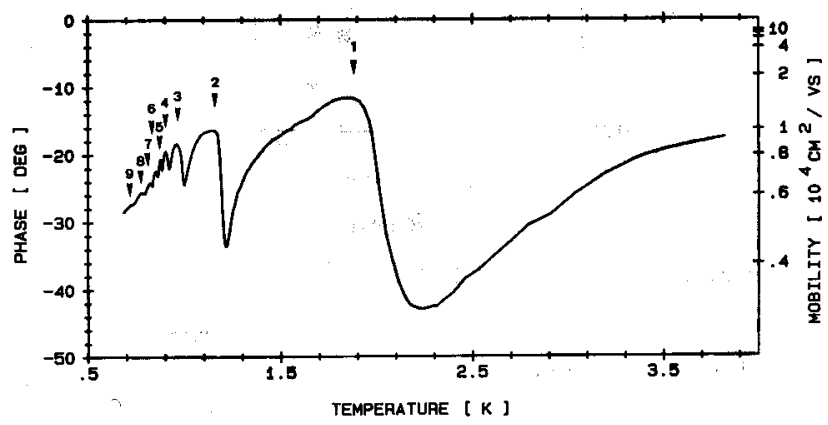

Fig. 1. Phase shift and mobility for electrons on solid hydrogen covered by a ${ }^{4}$ He film with a temperature-dependent thickness. 
this case is that the mobility varies smoothly with increasing film thickness to a thickness which can be considered bulk helium. Similar results were obtained with ${ }^{3} \mathrm{He}$.

\section{Discussion}

Earlier results by Paalanen and Iye /7/ exhibited four mobility oscillations when helium films were deposited at constant temperature. The first two oscillations were explained by scattering from lateral excitations in atoms filling the first monolayer, which is assumed to solidify, and subsequently the first normal fluid layer. The scattering amplitude is inversely proportional to the compressibility which has a minimum for filled monolayers. We interpret the additional oscillations as due to scattering from lateral density fluctuations in the uppermost unfilled superfluid layer. This contribution to the excitation spectrum of thin films, which is usually ignored, was discussed by Puff and Dash /8/.

Layering in thin films is exhibited by oscillations in the structure factor in a direction normal to the film. This quantity has been studied for very thin helium films with

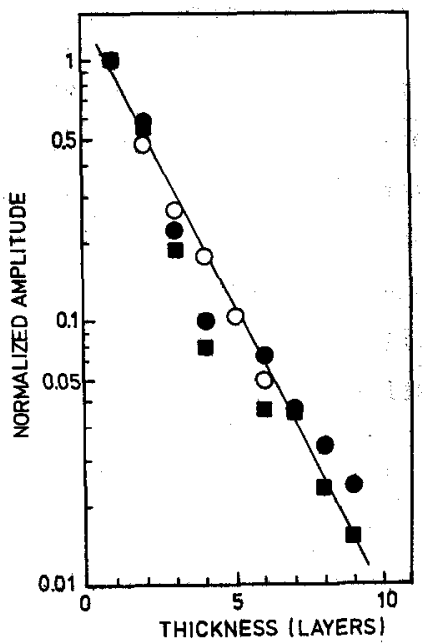

Fig. 2. Full symbols: Normalized amplitude of the modulation in the mobility as a function of the film thickness for two samples. Open symbols: Normalized amplitude of the oscillations in the density distribution of a Lennard-Jones system as one moves from the solid into the liquid phase (after Broughton et al. (10/). The straight line represents an exponential decay of the amplitudes with a characteristic length of 2.5 layers. computer simulations by Krotscheck $/ 9 /$. The amplitude of our mobility oscillations decays nearly exponentially with a characteristic length of about two layers. In our interpretation this variation of the amplitude is related to the decay of oscillations in the structure factor. In Fig. 2 the normalized amplitude of the mobility modulations is plotted versus layer number and compared with computer simulation results of the amplitude of the density modulation near a van der Waals solid-1iquid interface $/ 10 /$.

Our results are qualitatively consistent with scattering from lateral density fluctuations in the uppermost unfilled helium film layer. They imply layerwise adsorption of the superfluid film up to nine layers. The variation of the signal amplitude is similar to the decay of density correlations near a solid-liquid interface. We interpret the smooth variation of the electron scattering on thicker films as an indication of complete wetting of helium on hydrogen.

\section{Acknowledgements}

This work was supported in part by the Deutsche Forschungsgemeinschaft. One of us (A.J.D. ) was a Fulbright Fellow when this work was begun and received support from National Science Foundation Grant \# 82-13581.

References

1) J. Krim and J. G. Dash, Surf. Sci. 162 (1985) 421.

2) A. D. Migone, J. Krim and J. G. Dash, Phys. Rev. B 31 (1985) 7643 and references 5-8 therein.

3) S. Kumar and J. D. Maynard, to be published.

4) P. Taborek and L. Senator, Phys. Rev. Lett. 57 (1986) 218

5) D. Cieslikowski, A. J. Dahm, and P. Leiderer, Phys. Rev. Lett. 58 (1987) 1751.

6) R. Mehrotra and A. J. Dahm, J. of Low Temp. Phys. 67 (1987) 115.

7) M. A. Paalanen and Y. Iye, Phys. Rev. Lett. 55 (1985) 1761.

8) R. D. Puff and J. G. Dash, Phys. Rev. B 21 (1980) 2815 .

9) E. Krotscheck, Phys. Rev. B 32 (1985) 5713. 10) J. Q. Broughton, A. Bonissent, and F. F. Abraham, J. Chem. Phys. 74 (1981) 4029. 